\title{
Sonographic Evaluation of Chronic Kidney Disease Correlating with Serum Creatinine Level
}

\author{
Sidra Asif* \\ Doctor of Medical Imaging Technology, Department of Allied Health Sciences, Superior College Lahore, \\ University Campus, 17-KM Raiwaind Road, Kot Arain, Lahore, Pakistan \\ Sahar Khalil \\ Doctor of Medical Imaging Technology, Department of Allied Health Sciences, Superior College Lahore, \\ University Campus, 17-KM Raiwaind Road, Kot Arain, Lahore, Pakistan
}

Sybil Rose

Masters in Diagnostic Ultrasound, Lecturer, Department of Allied Health Sciences, Superior College Lahore, University Campus, 17-KM Raiwaind Road, Kot Arain, Lahore, Pakistan

Mussarat Ahmed

MBBS, DMRD, Consultant Radiologist, Life Care Hospital, Lahore, Pakistan

Rana Muhammad Athar Azeem Shams

Masters in Medical Imaging Technology, Lecturer, Department of Allied Health Sciences, Superior College Lahore, University Campus, 17-KM Raiwaind Road, Kot Arain, Lahore, Pakistan

Rana Muhammad Bakhtawar Khan Sajawal

Masters in Mathematics, Lecturer, Department of Allied Health Sciences, Superior College Lahore, University Campus, 17-KM Raiwaind Road, Kot Arain, Lahore, Pakistan

Ambreen Sadaf

Masters in Clinical Psychology, Lecturer, The University of Lahore, 1-KM Defence Road, near Bhuptian Chowk, Lahore, Pakistan

The research is financed by Asian Development Bank. No. 2006-A171(Sponsoring information)

\section{Abstract}

Background: Chronic kidney disease is defined as abnormality of urinary system, there is persistent abnormality of structure and upward renal excretory function that is sign of irreversible damage to function of nephron. It is a public health problem world widely and it is $12^{\text {th }}$ most common cause of mortality and morbidity, respectively.

Objective: To evaluate chronic kidney disease (CKD) sonographically in addition correlating echogenicity with the level of serum creatinine.

Study Design: An analytical Cross sectional prospective study.

Settings: Life Care Hospital, Radiology department Lahore.

Period: $20^{\text {th }}$ December 2020 till $10^{\text {th }}$ April 2021.

Material \& Methods: In our study all those patients with age above 18, patients suffering from chronic kidney disease, patient on hemodialysis, diabetic and hypertensive were included. While patient below 18 age, patients on peritoneal dialysis were excluded in which 71 patients were enrolled in the research. All the patient's data had been composed from indoor of hospital, outdoor of hospital, and emergency department of Life Care Hospital, Lahore. After informed consent, data was composed through ultrasound machine Toshiba Xario Prime.

Results: The findings revealed that there were 71 Chronic Renal Failure patients, 45 of whom were male and 26 of whom were female, with ages ranging from 20 to 73 . Since the $\mathrm{P}$ value is $>0.05$ of the relevance indicators, there is no important relationship between the two variables.

Conclusion: The ideal sonographic parameter that can be used to correlate the level of serum creatinine with kidney parameter within ultrasound is kidney cortical echogenicity. As kidney cortical echogenicity does not reverses as the disease progress even after the treatment, so it is the most accurate parameter that can be used.

Keywords: Chronic kidney disease, Echogenicity grade, Serum creatinine, Ultrasound, estimated Glomerular Filtration rate.

DOI: $10.7176 / \mathrm{JHMN} / 90-05$

Publication date:June $30^{\text {th }} 2021$

\section{Introduction}

Chronic kidney disease is defined as abnormality of urinary system, there is persistent abnormality of structure 
and upward renal excretory function that is sign of irreversible damage to function of nephron. It is a public health problem world widely and it is $12^{\text {th }}$ most common cause of mortality and morbidity, respectively. ${ }^{1} \mathrm{CKD}$ is identified as abnormal kidney structure that lasts longer than three months and has health consequences. In previous studies, there was no categorized data on renal cortical echogenicity in chronic kidney disease (CKD) and correlating with level of serum creatinine. So, to demonstrate the length of kidney, thickness of kidney, and echogenicity of parenchyma of kidney rather than from its significance in particularizing an enlarged collecting system recognizing the degree of damage in parenchyma of kidney and the probability of being reversed. In our study the sonographic evaluation will be done to associate the level of kidney function with systemic complications that develop during Chronic Kidney Disease.

CKD is identified as abnormal kidney structure that lasts longer than three months and has health consequences. In India, the prevalence of chronic kidney disease is approximately 800 and incidence occurs about 150-200 per million in population. ${ }^{1}$ The prevalence in Nepal is $10.6 \%$ and in Pakistan it is $23.3 \%$. In Asia, Sub Sahara Africa and India, people with CKD to glomerulonephritis and other unknown cause are more common. ${ }^{2}$ After the CKD has been confirmed, next step is to diagnose the stage of disease, cause of CKD must be identified, and must diagnose comorbid situations. ${ }^{3}$ Chronic kidney disease can be diagnosed by ultrasound or by kidney function markers such as blood urea nitrogen (BUN) or creatinine in blood or urine. ${ }^{5}$ Disease of kidney parenchyma can disturb either one of both of the kidneys could be a result of scarring and tissue damage. Ultrasound is a noninvasive and inexpensive technique and one of noninvasive technique imaging modality in radiology which do not use radiation and contrast medium to the patient. ${ }^{6}$ Ultrasound is real time imaging modality that can be used to demonstrate renal echogenicity and parameters. Because of its non-invasive nature and ease of access and visualization of the kidneys, the best imaging modality for CKD is ultrasonography. Most of the cases, main imaging examination needed in evaluation of chronic kidney failure is ultrasonography. Increase echogenicity of cortex within small kidney is a sign of irreversible damage. ${ }^{7}$ The most communal origin of chronic kidney disease is diabetic nephropathy. ${ }^{8}$ Patient with chronic kidney disease has atrophic kidney with increased renal echogenicity that can be differentiated on ultrasound with acute kidney disease. ${ }^{9}$

The purpose of our study was to evaluate chronic kidney disease (CKD) sonographically in addition correlating echogenicity with the level of serum creatinine. The serum creatinine level is a parameter for function of kidney but still instead of that renal echogenicity is best indicator to evaluate the function of kidney because after the therapy of kidney, serum creatinine improves over the period. A further complete study requires to be examined with additional generalizability by increasing sample size and study settings.

\subsection{Materials and Methods}

An analytical-cross sectional study was performed. The study was conducted in Life Care Hospital, Radiology Department Lahore, Punjab. 4 months after the approval of synopsis from $20^{\text {th }}$ December 2020 till $10^{\text {th }}$ April 2021. 71 out of which 45 were males and 26 were female. Non-Probability Convenient Sampling Technique was used. All those who had diabetes, duration of hypertension, other causes of chronic renal failure, treatment history will be collected, male and female and Age above $>18$ were included in our study. Patient with renal replacement therapy, Peritoneal dialysis and Renal transplant were excluded. Serum creatinine level was checked, and then transabdominal ultrasound was performed by using 3.5 -5 MHz convex transducer.

Renal dimensions, images were acquired in the longitudinal plane with both renal poles clearly demonstrated and on the transverse plane at the level of the hilum. Using electronic calipers, the length of kidney (L) was taken as the longest distance among the renal poles on longitudinal scan and the renal width (W) as the maximum transverse diameter on the transverse scan. The renal thickness or depth (D) was taken as the average of the maximum distance between the anterior and posterior walls of the mid portion of the kidney in the longitudinal and transverse scans (D1 and D2). The kidney volume was obtained using the prolate ellipsoid formula $(\mathrm{L} \times \mathrm{W} \times$ $\mathrm{D} 1+\mathrm{D} 2 / 2 \times 0.523)$. Cortical thickness was assessed on a longitudinal scan as the perpendicular distance from the base of a pyramid to the renal capsule, $2 \mathrm{~cm}$ away from the renal poles and at the mid portion of the kidney. The renal parenchymal volume was obtained by using longitudinal and transverse scans.

For age, average thickness of parenchyma, average longitudinal size, frequency, and percentages were calculated; for gender and echogenicity grade, average thickness of parenchyma, average size of longitudinal of kidney, frequency, and percentages were evaluated. One direction measurement of variance was used to measure the statistical analysis (ANOVA). Correlation coefficient analysis was used to determine the relationship between serum creatinine and sonographic parameters. Statistically significant difference was described as a $P$ value of less than 0.05 . The rules and regulations set by the ethical committee of The Superior College, Lahore were followed while conducting the research and the rights of the research participants were respected. Written informed consent attached was taken from all the participants.

\section{Results}

A total of 71 patients in which 45 were males and 26 were females with Chronic Kidney Disease. The patients 
were 47.4912 .31 years old on average. Below is a graph of the average of the serum creatinine, thickness of parenchyma, longitudinal length of kidney, and thickness of cortex.

\section{Discussion}

Out of 71 patients that are suffering from chronic kidney disease, 45 patients were male, and 26 patients were female. The age group was between 20 till 73 . Our study suggested that $75 \%$ of the patient are hypertensive, and $25 \%$ of patients are non-hypertensive but suffering from diabetes. The most common cause of chronic kidney disease among these groups was hypertension and second most common cause after hypertension was diabetes. Glodny B, et al., in their study "ultrasonography patterns for Hypertensive patients," they found that kidney change is easily detected in patients with hypertension by ultrasound as disease progresses.

Chronic kidney disease (CKD) is defined as progressive kidney damage that is irreversible, it is caused by destruction of both kidney function and structure that deteriorates with time. As the disease progresses, the problem is getting worse and both kidneys do not work efficiently. Its eithers affect GFR or not in some. Our study evaluates the functional capacity of both kidneys by comparing serum creatinine level with renal parameters in patients with Chronic Kidney Disease. Ultrasound is an ideal imaging modality, easily available and cheap. It is a real time imaging modality that can provide more accurate information as compared to others. Renal ultrasound parameters for instance longitudinal length of kidney, echogenicity of kidney, parenchyma, and thickness of cortex of kidney could be pretentious in chronic kidney disease (CKD).

Alsafi Ahmed Abdella et al., in their study, "ultrasound finding of renal failure patients and creatinine Serum level relationship" there study revealed that the volume of kidney decreases with increase the serum creatinine level. According to this study, there is no correlation between echogenicity grade and cortical thickness among the patients suffering from chronic renal failure because $p$ value is 131 , which is higher than the degree of importance. 05, indicating that the two variables have no meaningful relationship. As a result, the alternative hypothesis was accepted $\left(\mathrm{H}_{1}\right.$ : There seems to be no ultrasound association between thickness of cortex along kidney and kidney echogenicity in patients with chronic renal failure). In previous study by Michael D. Beland et.al., results showed that the average cortical thickness was $5.9 \mathrm{~mm}$ (range, $3.2-11.0 \mathrm{~mm}$ ); average length was $10 \mathrm{~cm}(7.2-12.4$ $\mathrm{cm})$; average minimum serum creatinine was $2.1 \mathrm{mg} / \mathrm{dL}(1.1-6.1 \mathrm{mg} / \mathrm{dL})$, and average glomerular filtration rate (eGFR) was $34.8 \mathrm{~mL} / \mathrm{min}(10.6-99.4 \mathrm{~mL} / \mathrm{min})$. There was a statistically significant relationship between eGFR and cortical thickness $(\mathrm{p}<0.0001)$. There was no statistically significant relationship between renal echogenicity and cortical thickness as $(\mathrm{p}=0.08)$. A study that was conducted on the topic "evaluation of renal changes in diabetic and hypertensive patients using ultrasound and laboratory findings". It also supports the present findings in the study that concluded that there is no significant sonographical correlation was observe between cortical thickness, cortical echogenicity, and cortico-medullary differentiation (CMD). Estimated glomerular filtration level (GFR) was the accurate lab investigation to identify and classify the loss of renal function. Serum creatinine level played an important role in the calculation of the estimated glomerular filtration level (eGFR). We cannot rely on blood urea nitrogen (BUN) alone. In the detection and identification of renal functional loss because it has to rely on different factors. Micro-albumin urea is an accurate lab test that detects small amount of albumin (protein) in urine, and it considers as early sign of renal function loss. In conclusion, lab investigations have superiority to ultrasound in detection and classification of renal function loss and concluded that the patients with chronic renal failure the cortical echogenicity increases while decreasing the renal cortical thickness.

The limitation of our study is that in this study group included more patients with Grade 3 and Grade 4 in comparison to patients with Grade 1 and Grade 2 with patients suffering from Chronic Kidney Disease. This most probably occurred due to the study that is directed in a care hospital of area. In this hospital mostly cases were cured with replacement of kidney like hemodialysis. Patients with transplantation of kidney along with peritoneal dialysis were omitted from our study.

\section{Conclusion}

The ideal sonographic parameter that can be used to correlate the level of serum creatinine with kidney parameter within ultrasound is kidney cortical echogenicity. As kidney cortical echogenicity does not reverses as the disease progress even after the treatment, so it is the most accurate parameter that can be used.

\section{Conflict of Interest}

There is no conflict of interest in this research.

\section{References}

1. Singh A, Gupta K, Chander R, Vira M. Sonographic Grading of Renal Cortical Echogenicity and Raised Serum Creatinine in Patients with Chronic Kidney Disease. Journal of Evolution of Medical and Dental Sciences. 2016 May 12;5(38):2279-87.

2. Webster Ac, Nagler Ev, Morton Rl, Masson P. Chronic Kidney Disease. The Lancet. 2017 Mar 


\section{5;389(10075):1238-52. Https://Doi.Org/10.1016/S0140-6736(16)32064-5}

3. Braconnier P, Piskunowicz M, Vakilzadeh N, Müller Me, Zürcher E, Burnier M, Pruijm M. How Reliable Is Renal Ultrasound To Measure Renal Length And Volume In Patients With Chronic Kidney Disease Compared With Magnetic Resonance Imaging?. Acta Radiologica. 2020 Jan;61(1):117-27. Doi/Full/10.1177/0284185119847680

4. Akbari A, Clase Cm, Acolt P, Battistella M, Bello A, Feltmate P, Grill A, Karanji M, Komenda P, Madore F, Manns Bj. Canadian Society of Nephrology Commentary on The Kdigo Clinical Practice Guideline for CKD Evaluation and Management. American Journal of Kidney Diseases. 2015 Feb 1;65(2):177-205. Https://Doi.Org/10.1053/J.Ajkd.2014.10.013

5. Hansen K1, Nielsen Mb, Ewertsen C. Ultrasonography of The Kidney: A Pictorial Review. Diagnostics. 2016 Mar;6(1):2. Https://Doi.Org/10.3390/Diagnostics6010002

6. Ahmed S, Bughio S, Hassan M, Lal S, Ali M. Role of Ultrasound in The Diagnosis of Chronic Kidney Disease and Its Correlation with Serum Creatinine Level. Cureus. 2019 Mar;11(3). Https://Dx.Doi.Org/10.7759\%2fcureus.4241

7. Nwafor Nn, Adeyekun Aa, Adenike Oa. Sonographic Evaluation of Renal Parameters in Individuals with Essential Hypertension and Correlation With Normotensives. Nigerian Journal of Clinical Practice. 2018;21(5):578-84. Https://Doi.Org/10.4103/Njcp.Njcp_57_17

8. Singh Aag, Kamlesh And Chander, Ramesh And Vira, Mayur. Sonographic Grading of Renal Cortical Echogenicity and Raised Serum Creatinine in Patients with Chronic Kidney Disease. Journal of Evolution of Medical and Dental Sciences. 2016;5(38):2279--87.

9. Asif Hs, Naeem Mw, Rose S, Hussain M, Iqbal R, Irfan M, Basheer M, Draz U, Malik Ss, Shams Rm, Farooq My. Ultrasonographic Correlation Of Cortical Thickness And Echogenicity Among Patients Suffering From Chronic Renal Failure. Journal Of Health And Medical Sciences. 2019 Oct 30;2(4). 10.31014/Aior.1994.02.04.71

10. Ahmed Sab, Sanobar And Hassan, Maria And Lal, Sajan And Ali, Muhammad. Role Of Ultrasound In The Diagnosis Of Chronic Kidney Disease And Its Correlation With Serum Creatinine Level. Cureus. 2019;11(3).

11. Mohammed, A. A. M. 2016. Evaluation Of Renal Changes In Diabetic And Hypertensive Patients Using Ultrasound And Laboratory Findings. Sudan University Of Science And Technology

12. Omer, M. A. A., Eljack, A. H., Gar-Alnabi, M. E., Mahmoud, M. Z., Elseid, M. \& Edam, G. A. 2014. Ultrasonographic Characteristics Of Diabetes Impacts In Kidneys' Morphology. Open Journal Of Radiology, 4,301 .

Table 1. Correlation of age, serum creatinine, left kidney longitudinal length, left kidney parenchymal thickness and cortical thickness.

\begin{tabular}{|l|l|l|l|l|l|l|}
\hline \multicolumn{2}{|c|}{} & Age & $\begin{array}{l}\text { Serum } \\
\text { Creatinine } \\
\text { (mg/dL) }\end{array}$ & $\begin{array}{l}\text { Left side Kidney } \\
\text { Longitudinal } \\
\text { length(cm) }\end{array}$ & $\begin{array}{l}\text { Left Kidney } \\
\text { Parenchymal } \\
\text { thickness(mm) }\end{array}$ & $\begin{array}{l}\text { Left side Kidney } \\
\text { thickness } \\
\text { cortex(mm) }\end{array}$ \\
\hline \multirow{2}{*}{$\mathrm{N}$} & Valid & 71 & 71 & 71 & 71 & 71 \\
\cline { 2 - 7 } & Missing & 0 & 0 & 0 & 0 & 0 \\
\hline Mean & 47.49 & 6.87 & 7.99 & 9.78 & 6.16 \\
\hline Median & 50 & 6.5 & 8.2 & 9.2 & 6.1 \\
\hline Std. Deviation & 12.315 & 2.493 & 1.425 & 2.354 & 0.898 \\
\hline \multirow{3}{*}{ Percentiles } & 25 & 37 & 5.2 & 7.2 & 8.3 & 5.6 \\
\cline { 2 - 7 } & 50 & 50 & 6.5 & 8.2 & 9.2 & 6.1 \\
\cline { 2 - 7 } & 75 & 56 & 8.7 & 8.9 & 11 & 6.9 \\
\hline
\end{tabular}


Table 2. There was significant correlation among echogenicity grades 3 and 4 in right kidney (ANOVA F-value= 3.192; $\mathrm{p}=0.05$ ) and (ANOVA $\mathrm{F}=4.48 ; \mathrm{p}=005$ ).

\begin{tabular}{|l|l|l|l|l|l|l|}
\hline \multirow{2}{*}{ Right Kidney Echogenicity grade 0 } & Between Groups & 0 & 48 & 0 &. &. \\
\cline { 2 - 7 } & Within Groups & 0 & 22 & 0 & & \\
\hline & Total & 0 & 70 & & & \\
\hline \multirow{4}{*}{ Right Kidney Echogenicity grade 1 } & Between Groups & 0 & 48 & 0 &. &. \\
\hline & Within Groups & 0 & 22 & 0 & & \\
\hline & Total & 0 & 70 & & & \\
\hline \multirow{4}{*}{ Right Kidney Echogenicity grade 2 } & Between Groups & 6.31 & 48 & 0.131 &. &. \\
\hline & Within Groups & 0 & 22 & 0 & & \\
\hline & Total & 6.31 & 70 & & & \\
\hline \multirow{3}{*}{ Right Kidney Echogenicity grade 3 } & Between Groups & 9.286 & 48 & 0.193 & 3.192 & 0.002 \\
\hline & Within Groups & 1.333 & 22 & 0.061 & & \\
\hline & Total & 10.62 & 70 & & & \\
\hline \multirow{3}{*}{ Right Kidney Echogenicity grade 4 } & Between Groups & 13.033 & 48 & 0.272 & 4.48 & 0 \\
\hline & Within Groups & 1.333 & 22 & 0.061 & & \\
\cline { 2 - 7 } & Total & 14.366 & 70 & & & \\
\hline
\end{tabular}

